\title{
Pulmonary Artery Sling, Congenital Blindness and Basal Ganglia Hemorrhage: A Fortuitous Association?
}

\author{
Mohammed Morjan ${ }^{\mathrm{a}, \mathrm{c}}$, Khaldoun Ali ${ }^{\mathrm{a}}$, Philipp Baumbach ${ }^{\mathrm{b}}$, Ingo Breitenbach ${ }^{\mathrm{a}}$, \\ Wolfgang Harringer ${ }^{\mathrm{a}}$, Aschraf El-Essawi ${ }^{\mathrm{a}}$
}

\begin{abstract}
Pulmonary artery sling (PAS) is a rare congenital vascular anomaly that typically presents in the first month of life with severe respiratory symptoms. A small subset of patients with this anomaly survives to adulthood with few or no symptoms. Several associated congenital anomalies were described. In this article, we describe an incidental finding of an asymptomatic PAS in an adult patient with congenital anisocoria and blindness 2 years after a spontaneous intracerebral hemorrhage. To the best of our knowledge, this is the first report on this association in an elderly patient. We believe that its documentation might contribute to a better understanding of this anomaly and its implications.
\end{abstract}

Keywords: Pulmonary artery sling; Basal ganglia hemorrhage

\section{Introduction}

Pulmonary artery sling (PAS) is a rare entity with a poorly defined embryology and natural history. In PAS, the left pulmonary artery typically arises anomalously from the posterior aspect of the right pulmonary artery, passes over the right main stem bronchus near its origin and courses leftward between the trachea and esophagus to the left pulmonary hilum [1]. No evidence of a genetic link to the presence of PAS has been documented and its embryology has not been

Manuscript accepted for publication December 9, 2013

\footnotetext{
a Department of Thoracic and Cardiovascular Surgery, Klinikum Braunschweig, Braunschweig, Germany

${ }^{\mathrm{b}}$ Department of Radiology, Klinikum Braunschweig, Braunschweig, Germany

${ }^{\mathrm{c} C}$ Corresponding author: Mohammed Morjan, Division of Cardiac, Thorax and Vascular Surgery, Klinikum Braunschweig, Salzdahlumer Strasse 90, Braunschweig 38126, Germany.

Email: Morjan79@gmail.com
}

doi: http://dx.doi.org/10.4021/jmc1617w completely delineated. However, PAS was described in association with the rare congenital syndrome of Mowat-Wilson, which includes pupillary abnormalities [2]. Spontaneous intracerebral hemorrhage (SIH) is a bleeding into the parenchyma of the brain that may extend into the ventricles and, in rare cases, the subarachnoid space. Although hypertensive intracerebral hemorrhage remains the most common form of intracerebral hemorrhage, underlying vascular abnormalities should always be considered [3]. An association between PAS and SIH has not yet been reported in the literature.

\section{Case Report}

A 68-year-old lady with congenital anisocoria and blindness was evaluated for an episode of atypical chest pain. The past medical history included hypertension and basal ganglia hemorrhage 2 years earlier with complete clinical recovery. Physical examination, electrocardiogram, routine laboratory investigations as well as trans-thoracic echocardiography revealed no abnormalities. Because of an enlarged mediastinal shadow on plain X-ray, the referring cardiologist sent the patient for a computed tomography (CT) scan of the chest. In the $\mathrm{CT}$, the left pulmonary artery was seen arising from the superior aspect of the right pulmonary artery and coursing

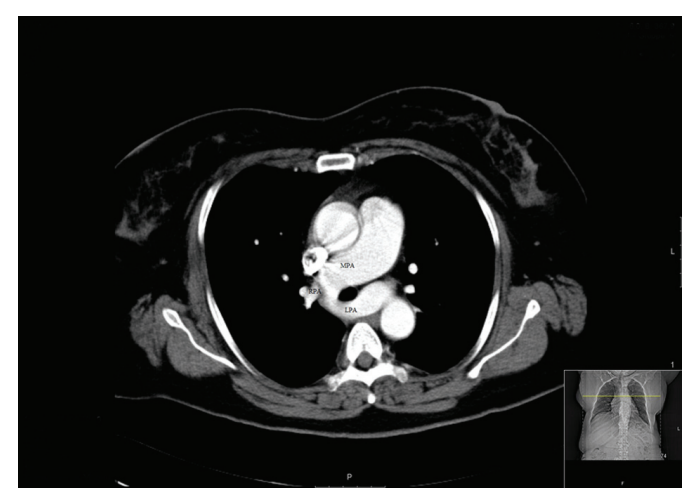

Figure 1. The left pulmonary artery was seen arising from the superior aspect of the right pulmonary artery and coursing posterior to the trachea. MPA: main pulmonary artery; LPA: left pulmonary artery; RPA: right pulmonary artery. 
posterior to the trachea at the level of the carina. No significant compressions of the main stem, right, or left bronchi were present, and no additional anomalies were observed (Fig. 1). The patient was transferred to our department. After 1 week of clinical observation and monitoring and in absence of symptoms, no surgical treatment was indicated and the patient was discharged. After 1 year the patient is in good conditions and free of any symptoms.

\section{Discussion}

The prevalence of PAS is purely conjectural, as there is no way to know the true number of asymptomatic cases that exist. In the known cases, PAS has been shown to have a male to female ratio of $3: 2$. The vast majority of patients have symptoms (90\%), and of those, $90 \%$ present in the first year of life [4]. Asymptomatic pulmonary slings are typically diagnosed incidentally in adolescence or adulthood. Associated anomalies of the tracheobronchial tree are frequent $(51 \%)$ while major cardiovascular abnormality are present in up to $30 \%$ of patients [5]. To date no case of simultaneous presence of SIH and PAS is described in literature. Hypertension is the most important risk factor for SIH. Other less well established risk factors include genetic factors such as mutations in the genes encoding the $a$-subunit of factor XIII. In the presence of SIH, underlying vascular abnormalities should always be considered, while association with extracranial vascular abnormalities cannot be excluded [3].

Until recently, no evidence of a genetic link to the presence of PAS has been documented. Mowat-Wilson syndrome is a congenital anomaly-mental retardation syndrome associated with PAS. A mosaic of eye defects has been reported in association with this syndrome. Haploinsufficiency of a gene termed ZFHX1B on chromosome 2 is responsible for this condition. The presence of pupillary abnormalities and congenital blindness in addition to the pulmonary sling in this patient raises suspicions of a masquerade congenital syndrome.

\section{Conclusion}

The case described in this article might be of help to better define the incidence and prevalence of pulmonary sling in adulthood. The unique association with the abnormalities described above reinforces the theory of a genetic link to the presence of PAS. We hope that our report can contribute to a better understanding of this congenital anomaly and raise awareness to the possibility of an association with SIH.

\section{Disclosure}

The authors report no conflict of interest.

\section{References}

1. Glaevecke H, Doehle W. Uber eine selten angeborene anomalie der pulmonalarterie. Munch Med Wochenschr.1897;44:950-953.

2. Adam MP, Schelley S, Gallagher R, Brady AN, Barr K, Blumberg B, Shieh JT, et al. Clinical features and management issues in Mowat-Wilson syndrome. Am J Med Genet A. 2006;140(24):2730-2741.

3. Qureshi AI, Tuhrim S, Broderick JP, Batjer HH, Hondo $\mathrm{H}$, Hanley DF. Spontaneous intracerebral hemorrhage. N Engl J Med. 2001;344(19):1450-1460.

4. Gikonyo BM, Jue KL, Edwards JE. Pulmonary vascular sling: report of seven cases and review of the literature. Pediatr Cardiol. 1989;10(2):81-89.

5. Dupuis C, Vaksmann G, Pernot C, Gerard R, Martinez J, Van Egmond H. Asymptomatic form of left pulmonary artery sling. Am J Cardiol. 1988;61(1):177-181. 